\title{
Imaging of magnetic nanoparticles by atomic magnetometers
}

\author{
V. Lebedev, N. Castagna, A. Weis \\ Physics Department, University of Fribourg, Fribourg, Switzerland, victor.lebedev@unifr.ch \\ B. Michen, A. Fink \\ Adolphe Merkle Institute, University of Fribourg, Marly, Switzerland \\ G. Bison \\ Biomagnetisches Zentrum, Universitätsklinikum Jena, Jena, Germany
}

\section{Introduction}

The imaging of the magnetic field from magnetic nanoparticles (MNP) implanted in biological tissues is becoming a novel technique for inferring information about the morphology of substructures, such as organs, tumors or cells. We study magneto-relaxometry (MRX), in which the relaxation time of MNPs yields information on the particles' environment. So far only liquid He-cooled SQUID magnetometers have been used for MRX measurements. Here, we propose two novel approaches to MRX-imaging.

\section{Methods}

MNP distributions of several $10 \mathrm{~cm}^{2}$ are recorded by an array of 19 Cs magnetometers using magnetic resonance. The device detects the temporal evolution of field maps from MNPs, functionalized to interact with a particular type of tissue or cells. For resolving MNP distributions on the sub-mm scale, we are currently developing a "magnetic field imaging camera" using Cs atoms contained in a cubic glass cell with inert buffer gas. A laser-based pump-probe scheme with CCD camera detection yields directly the MNP distribution, each pixel acting as an individual magnetometer. We anticipate a detection limit of $10 \mathrm{pT}$ with sub-mm spatial and a few ms time resolution.

\section{Results}

We have observed first MRX signals from immobilized MNPs using a single sensor of our array with pT sensitivity. We have built a prototype of the imaging camera device and present results of a space- and time-resolved study of atomic diffusion in a buffer gas.

\section{Conclusion}

We present prototypes of novel magnetic imaging systems for detecting large- and small-scale distributions of MNPs using MRX signals recorded by atomic magnetometers operated at room temperature. These systems offer distinct advantages (cost, logistics, sensor positioning) compared to the traditionally used cryogenic SQUID detectors. The high magnetometric sensitivity, spatial resolution and bandwidth of these devices give them a large potential for the efficient imaging of nanoscopic magnetic probes in biological samples. 